\begin{tabular}{|c|l|}
\hline Title & $\begin{array}{l}\text { Second-order Moller-Plesset perturbation (MP2) theory at finite temperature: relation with Surjan's density matrix MP2 } \\
\text { and its application to linear-scaling divide and-conquer method }\end{array}$ \\
\hline Author(s) & Kobay ashi, Masato; Taketsugu, Tetsuya \\
\hline Citation & $\begin{array}{l}\text { Theoretical chemistry accounts, 134(9), 107 } \\
\text { https://doi.org/10.1007/s00214_015-1710-y }\end{array}$ \\
\hline Issue Date & 2015-08-16 \\
\hline Doc URL & http://hdl.handle.net/2115/62731 \\
\hline Type & article(author version) \\
\hline File Information & TCA_DC-fracMP2_revise.pdf \\
\hline
\end{tabular}

Instructions for use 


\title{
Second-order Møller-Plesset perturbation (MP2) theory at finite temperature: Relation with Surján's density matrix MP2 and its application to linear-scaling divide-and-conquer method
}

\author{
Masato Kobayashi · Tetsuya Taketsugu
}

July 23, 2015

\begin{abstract}
In 2005, Surján showed two explicit formulas for evaluating the second-order Møller-Plesset perturbation (MP2) energy as a functional of the Hartree-Fock density matrix $D$ [Surján PR (2005) Chem Phys Lett 406:318], which are referred to as the $\Delta E_{\mathrm{MP} 2}[\boldsymbol{D}]$ functionals. In this paper, we present the finite-temperature (FT) MP2 energy functionals of the FT Hartree-Fock density matrix. There are also two formulas for the FT-MP2, namely, the conventional and renormalized ones, the latter of which has recently been formulated by Hirata and $\mathrm{He}$ [Hirata $\mathrm{S}, \mathrm{He}$ X (2013) J Chem Phys 138:204112]. We proved that there exists one-to-one correspondence between the formulas of two FT-MP2 and the $\Delta E_{\mathrm{MP} 2}[\boldsymbol{D}]$ functionals. This fact can explain the different behavior of two $\Delta E_{\mathrm{MP} 2}[\boldsymbol{D}]$ functionals when an approximate Hartree-Fock density matrix is applied, which was previously investigated by the authors [Kobayashi M, Nakai H (2006) Chem Phys Lett 420:250]. We also applied the FT-MP2 formalisms to the linear-scaling divide-and-conquer method for improving the accuracy with tiny addition of the computational efforts.
\end{abstract}

Keywords Fractional occupation number · Many-body perturbation theory · Laplace-transformed Møller-Plesset perturbation - Linear-scaling electronic structure method

This article is dedicated to Prof. Péter R. Surján on the occasion of his 60th birthday.

M. Kobayashi · T. Taketsugu

Department of Chemistry, Faculty of Science, Hokkaido University, Sapporo 060-0810, Japan

E-mail: k-masato@mail.sci.hokudai.ac.jp

E-mail: take@sci.hokudai.ac.jp

M. Kobayashi · T. Taketsugu

Elements Strategy Initiative for Catalysts and Batteries (ESICB), Kyoto University, Kyoto 615-8245, Japan

\section{Introduction}

The perturbation theory has widely been used in quantum chemistry to account for the dynamical electron correlation in single Slater [i.e., Hartree-Fock (HF)] and multideterminantal states [1]. Surján has worked on the perturbation theories for both HF and non-HF references. For non-HF reference functions, he and his coworkers proposed a series of multiconfiguration perturbation (MCPT) theories [2-6]. Because the MCPT theories are applicable to any reference functions, they have occasionally been applied to the antisymmetric product of strongly-orthogonal geminals wave functions [7-9].

Although many of Surján's famous works are related to the multideterminantal framework, he has also worked on the Møller-Plesset perturbation (MP) theory [10,11], where the HF wave function is adopted as the reference function. The importance of the MP theory, especially the secondorder MP (MP2) method, remains unchanged as the simplest non-empirical way to consider the electron correlation, even though the practical density functional theory (DFT) is getting mature $[12,13]$. The computational time for the canonical MP2 calculation, however, scales as $\mathcal{O}\left(N^{5}\right)$, where $N$ is the number of basis functions, which is significantly longer than those for the DFT and HF calculations of $\mathcal{O}\left(N^{3}\right)$. There have been proposed several techniques to reduce the computational time for the MP2 calculation. Surján [14] proposed a method to evaluate the MP2 correlation energy as the functional of the HF density matrix (DM), $D$, based on the Laplace-transformed MP2 formalism [15-17]. One of the authors (MK) followed this DM-MP2 or $\Delta E_{\mathrm{MP} 2}[\boldsymbol{D}]$ functional [18]. Its extension to higher-order MP energies was also mentioned by Surján and Szabados [19].

Another simple but straightforward way of reducing the computational time of quantum chemical calculations is the fragmentation of the system under consideration. 
Kobayashi, Nakai, and coworkers have developed one of them, called the divide-and-conquer (DC) method [20-22], which was first proposed by Yang [23,24] in the framework of the mean-field theories. The characteristic feature of the DC method, which allows the method to be applied to delocalized systems, is the use of the overlapped fragmentation that is managed by introducing the buffer region with the assistance of the finite-temperature (FT) HF formalism. They also proposed two types of the extension of the DC method to the post-HF correlation theories, including MP2. The first one, called the DC-DM MP2 method, applies the approximate $\mathrm{HF}$ density matrix obtained from the DC-HF calculation to the $\Delta E_{\mathrm{MP} 2}[\boldsymbol{D}]$ functional [25]. In the other method, called the DC-MP2 method, the correlation energy corresponding to each subsystem is evaluated using the subsystem molecular orbitals (MOs) [26]. The latter one has also been applied to the cluster expansion theories [27-29]. Although it was practically found that the DC-DM MP2 energy often shows better agreement with the canonical MP2 energy than the DC-MP2 one, the DC-MP2 method is usually adopted because of its smaller computational cost. In the DC-DM MP2 method, the FT effect is considered to be included through the use of FT DC-HF density matrix. But in the DC-MP2 method, the subsystem MOs are clearly separated into occupied and virtual ones.

By the way, the MP2 theory was also extended to the FT ensemble $[30,31]$ based on the FT Green's function theory. Recently, Hirata and He [32] formulated a novel representation, called the renormalized formula, which is free from the so-called Kohn-Luttinger conundrum [33]. Kohn-Luttinger conundrum refers to the following inconsistency: taking the limit of $T \rightarrow 0$ for the conventional FT many-body perturbation formalism does not lead to the zero-temperature correspondence for metallic system because of the appearance of the anomalous diagram. In addition, the renormalized FTMP2 method consistently connects the divergence rates of the zero-temperature MP2 energy for homogeneous electron gas system to the FT formalism. Their method is based on the FT normal ordering and thermal Wick's theorem [34, 35]. In this paper, we reveal the relation between two DMMP2 and two FT-MP2 energy expressions. Then, we introduce the FT effect to the DC-MP2 correlation energy. The theoretical aspects of this paper are given in Sect. 2. It is followed by the numerical assessment in calculations of small benzene molecule and fairly large polyene system, $\mathrm{C}_{60} \mathrm{H}_{62}$.

\section{Theory}

\subsection{Laplace-transformed MP2 and $\Delta E_{\mathrm{MP} 2}[\boldsymbol{D}]$ functionals}

The closed-shell pure-state (i.e., zero temperature) MP2 correlation energy is expressed by $[10,11,36]$

$\Delta E_{\mathrm{MP} 2}=\sum_{i j}^{\mathrm{occ}} \sum_{a b}^{\mathrm{vir}}\langle i j \mid a b\rangle\left(2 \tilde{t}_{i j, a b}-\tilde{t}_{i j, b a}\right)$,

with the following amplitude

$\tilde{t}_{i j, a b}=-\frac{\langle a b \mid i j\rangle}{\varepsilon_{a}+\varepsilon_{b}-\varepsilon_{i}-\varepsilon_{j}}$.

Through this paper, $\{i, j, \cdots\}$ and $\{a, b, \cdots\}$ refer to occupied and virtual MOs for pure HF state, respectively, and $\{p, q, \cdots\}$ to arbitrary MOs, which are constructed as the linear combination of atomic orbitals (AOs), $\left\{\phi_{\mu}\right\}$,

$\psi_{p}=\sum_{\mu} C_{\mu p} \phi_{\mu}$.

Here, $\mathbf{C}_{p}$ and $\varepsilon_{p}$ are the coefficient vector and the energy of the MO $p$, obtained by solving the following Roothaan equation:

$\boldsymbol{F} \mathbf{C}_{p}=\varepsilon_{p} S \mathbf{C}_{p}$

$\boldsymbol{F}$ and $\boldsymbol{S}$ are the Fock and overlap matrices, respectively, of which the elements are expressed by

$$
\begin{aligned}
& F_{\mu \nu}=H_{\mu \nu}^{\text {core }}+\sum_{\lambda \sigma} D_{\lambda \sigma}[2\langle\mu \sigma \mid \nu \lambda\rangle-\langle\mu \sigma \mid \lambda \nu\rangle], \\
& S_{\mu \nu}=\left\langle\phi_{\mu} \mid \phi_{\nu}\right\rangle, \\
& H_{\mu \nu}^{\text {core }}=\left\langle\phi_{\mu}|\hat{h}| \phi_{\nu}\right\rangle,
\end{aligned}
$$

with the usual two-electron integral notation of $\langle\mu \sigma \mid \nu \lambda\rangle=\iint \phi_{\mu}\left(\mathbf{r}_{1}\right) \phi_{\sigma}\left(\mathbf{r}_{2}\right) r_{12}^{-1} \phi_{\nu}\left(\mathbf{r}_{1}\right) \phi_{\lambda}\left(\mathbf{r}_{2}\right) d \mathbf{r}_{1} d \mathbf{r}_{2}$, the one-electron Hamiltonian of $\hat{h}$, and the HF density matrix $\boldsymbol{D}$ at zero temperature:

$\boldsymbol{D}=\sum_{i}^{\text {occ }} \mathbf{C}_{i} \mathbf{C}_{i}^{\mathrm{T}}$.

Due to the existence of the denominator in Eq. (2), the straightforward computation of the MP2 energy with Eq. (1) requires $\mathcal{O}\left(N^{5}\right)$ time with the number of basis functions $N$. Almlöf [15] first used the Laplace transformation for evaluating the MP2 energy to remove the denominator:

$\Delta E_{\mathrm{MP} 2}=-\int_{0}^{\infty} e_{2}(t) d t$.

The integrand in Eq. (9) is given in AO-based formalism by $[16,17]$

$$
\begin{array}{r}
e_{2}(t)=\sum_{\gamma \delta \kappa \varepsilon} \sum_{\mu \nu \lambda \sigma} X_{\mu \gamma}(t) Y_{\nu \delta}(t) X_{\lambda \kappa}(t) Y_{\sigma \varepsilon}(t) \\
\langle\gamma \kappa \mid \delta \varepsilon\rangle[2\langle\nu \sigma \mid \mu \lambda\rangle-\langle\nu \sigma \mid \lambda \mu\rangle],
\end{array}
$$


where $\boldsymbol{X}(t)$ and $\boldsymbol{Y}(t)$ are the energy-weighted density matrices of electron and hole given by

$\boldsymbol{X}(t)=\sum_{i}^{\text {occ }} e^{\varepsilon_{i} t} \mathbf{C}_{i} \mathbf{C}_{i}^{\mathrm{T}}$,

$\boldsymbol{Y}(t)=\sum_{a}^{\mathrm{vir}} e^{-\varepsilon_{a} t} \mathbf{C}_{a} \mathbf{C}_{a}^{\mathrm{T}}$,

respectively. Because these matrices are sparse for large systems, the evaluation of the MP2 energy can be reduced to $\mathcal{O}\left(N^{2}\right)$ [16] or even $\mathcal{O}(N)$ [17].

Using this Laplace MP2 formalism, Surján [14] derived the explicit MP2 energy functional of the HF density matrix, while Ayala and Scuseria [17] suggested previously. Using the Roothaan equation (4), one can transform Eqs. (11) and (12) to

$\boldsymbol{X}(t)=e^{t \boldsymbol{S}^{-1} \boldsymbol{F}} \boldsymbol{D}$,

$\boldsymbol{Y}(t)=e^{-t \boldsymbol{S}^{-1} \boldsymbol{F}} \overline{\boldsymbol{D}}$,

where $\bar{D}$ is the complement of the HF density matrix $\boldsymbol{D}$ :

$\overline{\boldsymbol{D}}=\sum_{a}^{\mathrm{vir}} \mathbf{C}_{a} \mathbf{C}_{a}^{\mathrm{T}}=\boldsymbol{S}^{-1}-\boldsymbol{D}$.

Substituting Eqs. (13) and (14) for Eq. (10), the MP2 correlation energy can be obtained as a functional of the $\mathrm{HF}$ density matrix, which is referred to as $\Delta E_{\mathrm{MP} 2}[\boldsymbol{D}]$ functional or DM-MP2 method in this paper. Hereafter, Eqs. (13) and (14) are referred to as the $\boldsymbol{S}^{-1} \boldsymbol{F}$ formulas. Furthermore, using the commutation relationship of $\boldsymbol{F} \boldsymbol{D} \boldsymbol{S}=\boldsymbol{S} \boldsymbol{D F}$ and the idempotency of the density matrix, $(\boldsymbol{D S})^{n}=\boldsymbol{D S}$, one can further derive

$\boldsymbol{X}(t)=e^{t \boldsymbol{D F}} \boldsymbol{D}$,

$\boldsymbol{Y}(t)=e^{-t \overline{\boldsymbol{D}} \boldsymbol{F}} \overline{\boldsymbol{D}}$.

These equations, referred to as the $\boldsymbol{D F}$ formulas, can also be used instead of Eqs. (13) and (14).

This $\Delta E_{\mathrm{MP} 2}[\boldsymbol{D}]$ functional can be used to obtain proper MP2 correlation energy from the non-canonical HF method that cannot yield MOs of the system. The performance of the DM-MP2 method for the use with approximate density matrix was numerically assessed by Kobayashi and Nakai [18]. As an example, the density matrix obtained from the DC-HF calculation was applied to this functional [25]. Although this scheme, called the DC-DM MP2 method, succeeded in accurately evaluating the MP2 correlation energy based on the DC method, the DC-MP2 method based on the subsystem MOs, explained in Sect. 2.3, has been used as the standard extension of the DC method to the MP2 theory.
2.2 Finite temperature MP2 and Laplace-transformed formula

For the FT ensemble, the HF density matrix is expressed as [37, 38]

$\boldsymbol{D}=\sum_{p} f_{p} \mathbf{C}_{p} \mathbf{C}_{p}^{\mathrm{T}}$

where $f_{p}$ is the Fermi-distributed occupation number,

$f_{p}=f_{\beta}\left(\varepsilon_{\mathrm{F}}-\varepsilon_{p}\right)$

with Fermi level, $\varepsilon_{\mathrm{F}}$, and Fermi distribution function $f_{\beta}(x)=[1+\exp (-\beta x)]^{-1}$ for the inverse temperature $\beta=\left(k_{\mathrm{B}} T\right)^{-1}$. The electronic FT-HF energy is expressed by the usual formula as

$E_{\mathrm{HF}}=\operatorname{Tr}\left[\boldsymbol{D}\left(\boldsymbol{H}^{\text {core }}+\boldsymbol{F}\right)\right]$,

with the FT density matrix of Eq. (18). Although the grand potential can also be evaluated with the entropy, obtained from the occupation numbers, and the Fermi level, we do not care about these terms in this study because they are not included in the DC formalism [20,24].

The MP2 theory has also been generalized to the FT ensemble $[31,38,39]$. At finite temperature, the MP2 correlation energy is conventionally expressed as

$\Delta E_{\mathrm{MP} 2}^{\mathrm{C}}=\sum_{p q r s}\langle p q \mid r s\rangle\left(2 \tilde{t}_{p q, r s}^{\mathrm{C}}-\tilde{t}_{p q, s r}^{\mathrm{C}}\right)$.

with the following amplitude

$\tilde{t}_{p q, r s}^{\mathrm{C}}=-\frac{f_{p q, r s}\langle r s \mid p q\rangle}{\varepsilon_{r}+\varepsilon_{s}-\varepsilon_{p}-\varepsilon_{q}}$.

Here, $f_{p q, r s}$ is obtained from the orbital occupation number of Eq. (19) and its complement,

$\bar{f}_{p}=1-f_{p}$,

by

$f_{p q, r s}=f_{p} f_{q} \bar{f}_{r} \bar{f}_{s}$.

The energy expression of Eq. (21) can be derived from the finite-temperature Green's function [40]. Obviously, Eq. (22) diverges at finite $\beta$ (or non-zero temperature) for $p=r$ and $q=s$, for example. These divergent terms should be neglected in practical calculations. Hirata and He [32] recently proposed the renormalized formalism for the FT many-body perturbation theory that changes the divergence rate to be consistent to the zero-temperature counterpart:

$$
\begin{aligned}
& \Delta E_{\mathrm{MP} 2}^{\mathrm{R}}=\sum_{p q r s}\langle p q \mid r s\rangle\left(2 \tilde{t}_{p q, r s}^{\mathrm{R}}-\tilde{t}_{p q, s r}^{\mathrm{R}}\right), \\
& \tilde{t}_{p q, r s}^{\mathrm{R}}=-\frac{f_{p q, r s}\langle r s \mid p q\rangle}{\bar{f}_{r} \varepsilon_{r}+\bar{f}_{s} \varepsilon_{s}-f_{p} \varepsilon_{p}-f_{q} \varepsilon_{q}} .
\end{aligned}
$$

This formula comes from the thermal Wick's theorem [34, 35]. Note that this renormalized FT-MP2 energy as well 
as the conventional FT-MP2 energy diverge for metallic system. The conventional FT-MP2 energy, however, may diverge even for insulators. In this subsection, we clarify the correspondence of the above two formalisms with two $\Delta E_{\mathrm{MP} 2}[\boldsymbol{D}]$ functionals.

According to the standard AO-based formalism [17], the Laplace transformation of $\Delta E_{\mathrm{MP} 2}^{\mathrm{C}}$ and $\Delta E_{\mathrm{MP} 2}^{\mathrm{R}}$ leads to

$\Delta E_{\mathrm{MP} 2}^{\mathrm{C} / \mathrm{R}}=-\int_{0}^{\infty} e_{2}^{\mathrm{C} / \mathrm{R}}(t) d t$

The integrand in Eq. (27) is given by

$$
\begin{array}{r}
e_{2}^{\mathrm{C} / \mathrm{R}}(t)=\sum_{\gamma \delta \kappa \varepsilon} \sum_{\mu \nu \lambda \sigma} X_{\mu \gamma}^{\mathrm{C} / \mathrm{R}}(t) Y_{\nu \delta}^{\mathrm{C} / \mathrm{R}}(t) X_{\lambda \kappa}^{\mathrm{C} / \mathrm{R}}(t) Y_{\sigma \varepsilon}^{\mathrm{C} / \mathrm{R}}(t) \\
\langle\gamma \kappa \mid \delta \varepsilon\rangle[2\langle\nu \sigma \mid \mu \lambda\rangle-\langle\nu \sigma \mid \lambda \mu\rangle] .
\end{array}
$$

For the conventional formalism, the energy-weighted density matrices are given by

$\boldsymbol{X}^{\mathrm{C}}(t)=\sum_{p} e^{\varepsilon_{p} t} f_{p} \mathbf{C}_{p} \mathbf{C}_{p}^{\mathrm{T}}$,

$\boldsymbol{Y}^{\mathrm{C}}(t)=\sum_{p} e^{-\varepsilon_{p} t} \bar{f}_{p} \mathbf{C}_{p} \mathbf{C}_{p}^{\mathrm{T}}$,

and for the renormalized formalism, they are

$\boldsymbol{X}^{\mathrm{R}}(t)=\sum_{p} e^{\varepsilon_{p} f_{p} t} f_{p} \mathbf{C}_{p} \mathbf{C}_{p}^{\mathrm{T}}$

$\boldsymbol{Y}^{\mathrm{R}}(t)=\sum_{p} e^{-\varepsilon_{p} \bar{f}_{p} t} \bar{f}_{p} \mathbf{C}_{p} \mathbf{C}_{p}^{\mathrm{T}}$

The expressions for the conventional formalism are different from Eqs. (11) and (12) only in the existence of the occupation number before $\mathbf{C}_{p} \mathbf{C}_{p}^{\mathrm{T}}$. For the renormalized formalism, the occupation number also appears on the exponential.

If the HF equation of Eq. (4) is satisfied, one can derive the following equation:

$\boldsymbol{X}^{\mathrm{C}}(t)=e^{t \boldsymbol{S}^{-1} \boldsymbol{F}} \boldsymbol{D}$,

$\boldsymbol{Y}^{\mathrm{C}}(t)=e^{-t \boldsymbol{S}^{-1} \boldsymbol{F}} \overline{\boldsymbol{D}}$,

where $\bar{D}$ is the complement of the FT-HF density matrix,

$\overline{\boldsymbol{D}}=\sum_{p} \bar{f}_{p} \mathbf{C}_{p} \mathbf{C}_{p}^{\mathrm{T}}=\boldsymbol{S}^{-1}-\boldsymbol{D}$

These are formally the same as the $\boldsymbol{S}^{-1} \boldsymbol{F}$ formulas of Eqs. (13) and (14). However, it cannot be further transformed to the $\boldsymbol{D F}$ formulas because the density matrix is no longer idempotent at finite temperature but has the following relationship:

$(\boldsymbol{D} \boldsymbol{S})^{n}=\sum_{p} f_{p}^{n} \mathbf{C}_{p} \mathbf{C}_{p}^{\mathrm{T}} \boldsymbol{S}$.

As for the renormalized formulas, on the contrary, using the relationships of $\boldsymbol{F} \boldsymbol{D} \boldsymbol{S}=\boldsymbol{S D F}$ and Eq. (36) leads to

$\boldsymbol{X}^{\mathrm{R}}(t)=e^{t \boldsymbol{D} \boldsymbol{F}} \boldsymbol{D}$,
$\boldsymbol{Y}^{\mathrm{R}}(t)=e^{-t \overline{\boldsymbol{D}} \boldsymbol{F}} \overline{\boldsymbol{D}}$

These are formally the same as the $\boldsymbol{D F}$ formulas of Eqs. (16) and (17).

Here, we summarize the important point in this Section. As derived in the previous studies [14,18], two DM-MP2 formalisms (i.e., the $\boldsymbol{S}^{-1} \boldsymbol{F}$ and $\boldsymbol{D F}$ formulas) are equivalent at zero temperature [see Eqs. (13) and (16), for example]. At finite temperature, however, these two formulas are no longer equivalent and further coincide with the conventional and renormalized FT-MP2 formulas [see Eqs. (33) and (37), for example].

When Kobayashi and Nakai [18] calculated the MP2 correlation energy using Eq. (10) with the density matrix having random noise, they obtained a reasonable MP2 correlation energy with Eqs. (16) and (17), although they could not with Eqs. (13) and (14). The noise-introduced HF density matrix can be represented as Eq. (18) having noise in the occupation number, $f_{p}$, if the basis set spans the complete space. Therefore, this result can now be interpreted that the amplitude of Eq. (22) for $p=r$ and $q=s$, for instance, diverges when using a density matrix for a mixed state.

\subsection{Finite temperature DC-MP2 method}

Kobayashi and coworkers [20,21,26,41] have proposed a linear-scaling DC-MP2 method. This method utilizes MOs determined in the subsystem $\alpha,\left\{\psi_{p}^{\alpha}\right\}$, which are constructed as

$\psi_{p}^{\alpha}=\sum_{\mu \in \mathcal{L}(\alpha)} C_{\mu p}^{\alpha} \phi_{\mu}$,

where the coefficient $C_{\mu p}^{\alpha}$ is obtained by solving the HF equation in subsystem $\alpha$ :

$\boldsymbol{F}^{\alpha} \mathbf{C}_{p}^{\alpha}=\varepsilon_{p}^{\alpha} \boldsymbol{S}^{\alpha} \mathbf{C}_{p}^{\alpha}$

In Eq. (39), $\mathcal{L}(\alpha)$ is the set of AOs used in the expansion of MOs in subsystem $\alpha$, which consists of two parts:

$\mathcal{L}(\alpha)=\mathcal{S}(\alpha) \sqcup \mathcal{B}(\alpha)$.

$\mathcal{S}(\alpha)$ is the AOs corresponding to the central region of the subsystem $\alpha$, which is mutually exclusive:

$\mathcal{S}(\alpha) \cap \mathcal{S}(\beta)=\emptyset, \forall \alpha \neq \beta$,

and $\mathcal{B}(\alpha)$ is the AOs corresponding to the neighboring region of the central region of the subsystem $\alpha$, called the buffer region. $\boldsymbol{F}^{\alpha}$ and $\boldsymbol{S}^{\alpha}$ are the subsystem Fock and overlap matrices, respectively, of which the elements are expressed by

$F_{\mu \nu}^{\alpha}=H_{\mu \nu}^{\mathrm{core}}+\sum_{\lambda \sigma} D_{\lambda \sigma}[2\langle\mu \sigma \mid \nu \lambda\rangle-\langle\mu \sigma \mid \lambda \nu\rangle]$

$S_{\mu \nu}^{\alpha}=\left\langle\phi_{\mu} \mid \phi_{\nu}\right\rangle$ 
Obviously, the matrix elements of Eqs. (43) and (44) are the same as Eqs. (5) and (6). Here in Eq. (43), one can adopt any density matrix, $\boldsymbol{D}$, which is appropriately determined. A possible candidate is the DC-HF density matrix:

$D_{\mu \nu}^{\mathrm{DC}}=\sum_{\alpha} P_{\mu \nu}^{\alpha} D_{\mu \nu}^{\alpha}$

where

$\boldsymbol{D}^{\alpha}=\sum_{p}^{\mathrm{MO}(\alpha)} f_{p}^{\alpha} \mathbf{C}_{p}^{\alpha} \mathbf{C}_{p}^{\alpha \mathrm{T}}$.

$f_{p}^{\alpha}$ is the Fermi-distributed occupation number:

$f_{p}^{\alpha}=f_{\beta}\left(\varepsilon_{\mathrm{F}}-\varepsilon_{p}^{\alpha}\right)$,

and $\boldsymbol{P}$ is the partition matrix defined by

$P_{\mu \nu}^{\alpha}= \begin{cases}1 & (\mu \in \mathcal{S}(\alpha) \wedge \nu \in \mathcal{S}(\alpha)) \\ 1 / 2 & (\mu \in \mathcal{S}(\alpha) \wedge \nu \in \mathcal{B}(\alpha), \text { or vice versa }) \\ 0 & \text { (otherwise). }\end{cases}$

Fermi level, $\varepsilon_{\mathrm{F}}$, in Eq. (47) is determined from the number of electrons in the entire system, $n_{\mathrm{e}}$, by solving the following equation:

$n_{\mathrm{e}}=\operatorname{tr}\left(\boldsymbol{D}^{\mathrm{DC}} \boldsymbol{S}\right)$.

As shown above, the Fermi level and the FT formalism are introduced in the DC-HF method. Recently, Yoshikawa and Nakai proposed an alternative DC procedure, where the number of electrons in each subsystem is fixed after a couple of iterations for improving the parallel efficiency of the DCHF calculations [42]. The Fermi level is no longer constant through the entire system in this scheme, which is also related to the adjustable density matrix assembly method [43, 44].

In the DC-MP2 method, the total correlation energy is estimated by summing up the subsystem correlation energies:

$\Delta E_{\mathrm{DC}-\mathrm{MP} 2}=\sum_{\alpha} \Delta E_{\mathrm{MP} 2}^{\alpha}$

The correlation energy of the subsystem $\alpha, \Delta E_{\mathrm{MP} 2}^{\alpha}$, has been estimated from the energy density analysis [45] with the subsystem MOs as follows:

$$
\begin{array}{r}
\Delta E_{\mathrm{MP} 2}^{\alpha}=\sum_{i j}^{\mathrm{occ}(\alpha)} \sum_{a b}^{\operatorname{vir}(\alpha)} \sum_{\mu \in \mathcal{S}(\alpha)} C_{\mu i}^{\alpha}\left\langle\mu j^{\alpha} \mid a^{\alpha} b^{\alpha}\right\rangle \\
\left(2 \tilde{t}_{i j, a b}^{\alpha}-\tilde{t}_{i j, b a}^{\alpha}\right) .
\end{array}
$$

$\tilde{t}_{i j, a b}^{\alpha}$ is the effective two-electron excitation amplitude for subsystem $\alpha$, which is expressed in MP2 case with the subsystem MOs as follows:

$\tilde{t}_{i j, a b}^{\alpha}=-\frac{\left\langle a^{\alpha} b^{\alpha} \mid i^{\alpha} j^{\alpha}\right\rangle}{\varepsilon_{a}^{\alpha}+\varepsilon_{b}^{\alpha}-\varepsilon_{i}^{\alpha}-\varepsilon_{j}^{\alpha}}$.

In the previous DC-MP2 method with integer occupation numbers, the subsystem MOs should clearly be separated into occupied and virtual ones by the Fermi level. In the DC-HF method, however, fractional occupations of MOs are allowed around Fermi level because the FT ensemble is formally treated (see Eq. (46)).

It is also possible to adopt the FT-MP2 formalisms of Eq. (21) or (25) in the DC-MP2 method. On the analogy to Eq. (51), the subsystem correlation energy at finite temperature can be evaluated as

$$
\begin{array}{r}
\Delta E_{\mathrm{MP} 2}^{\mathrm{C} / \mathrm{R} \alpha}=\sum_{p q r s}^{\mathrm{MO}(\alpha)} \sum_{\mu \in \mathcal{S}(\alpha)} C_{\mu p}^{\alpha}\left\langle\mu q^{\alpha} \mid r^{\alpha} s^{\alpha}\right\rangle \\
\left(2 \tilde{t}_{p q, r s}^{\mathrm{C} / \mathrm{R} \alpha}-\tilde{t}_{p q, r s}^{\mathrm{C} / \mathrm{R} \alpha}\right) .
\end{array}
$$

$\tilde{t}_{p q, r s}^{\mathrm{C} / \mathrm{R} \alpha}$ is

$\tilde{t}_{p q, r s}^{\mathrm{C} \alpha}=-\frac{f_{p q, r s}^{\alpha}\left\langle r^{\alpha} s^{\alpha} \mid p^{\alpha} q^{\alpha}\right\rangle}{\varepsilon_{r}^{\alpha}+\varepsilon_{s}^{\alpha}-\varepsilon_{p}^{\alpha}-\varepsilon_{q}^{\alpha}}$,

or

$\tilde{t}_{p q, r s}^{\mathrm{R} \alpha}=-\frac{f_{p q, r s}^{\alpha}\left\langle r^{\alpha} s^{\alpha} \mid p^{\alpha} q^{\alpha}\right\rangle}{\bar{f}_{r}^{\alpha} \varepsilon_{r}^{\alpha}+\bar{f}_{s}^{\alpha} \varepsilon_{s}^{\alpha}-f_{p}^{\alpha} \varepsilon_{p}^{\alpha}-f_{q}^{\alpha} \varepsilon_{q}^{\alpha}}$,

where

$\bar{f}_{p}^{\alpha}=1-f_{p}^{\alpha}$,

$f_{p q, r s}^{\alpha}=f_{p}^{\alpha} f_{q}^{\alpha} \bar{f}_{r}^{\alpha} \bar{f}_{s}^{\alpha}$.

As mentioned in the previous DC-MP2 and DC coupled cluster papers $[27,28,41,46,47]$, the buffer region used in the DC-MP2 method can be set to be smaller than that in the DC-HF method without significant loss of accuracy. When this dual-buffer DC-MP2 scheme was adopted, the Fermi level was used to be redetermined using the MOs constructed in the smaller subsystems. In the integer-occupation DC-MP2 method, however, the use of the Fermi level obtained from the prior DC-HF calculation does not significantly change the results because the Fermi level is used only for separating the subsystem MOs into occupied and virtual ones. On the other hand, in the FT DC-MP2 method, the subsystem MP2 correlation energy of Eq. (53) may largely depend on the Fermi level due to the occupation numbers appearing in the coefficients of Eqs. (54) and (55). Because the reference state for the DC-MP2 method is the preceding DC-HF state, the authors decided to use the Fermi level determined in the DC-HF calculation throughout the DC-MP2 calculation. The dependence of the FT DC-MP2 energy on the Fermi level will also be assessed below.

Fundamentally, the difference between the implementations of the FT and integer-occupation DC-MP2 methods is the summation lengths in Eqs. (51) and (53). Although the MO indices in Eq. (53) formally run over all subsystem orbitals, the MO $p$ of subsystem $\alpha$ is regarded as to be doublyoccupied or fully vacant if $f_{p}^{\alpha}>1-\theta$ or $f_{p}^{\alpha}<\theta$, respectively (throughout this paper, the threshold of $\theta=10^{-15}$ is adopted). Therefore, the computational costs for the MP2 
calculation of subsystem $\alpha$ depend on the practical numbers of occupied and virtual MOs, which are defined with the numbers of all MOs $\left(N^{\alpha}\right)$, uncorrelated core $\operatorname{MOs}\left(N_{\text {core }}^{\alpha}\right)$, doubly-occupied MOs $\left(N_{\text {docc }}^{\alpha}\right.$, including $N_{\text {core }}^{\alpha}$ ), and fullyvacant $\operatorname{MOs}\left(N_{\text {fvac }}^{\alpha}\right)$ by

$N_{\mathrm{occ}}^{\alpha}=N^{\alpha}-N_{\mathrm{fvac}}^{\alpha}-N_{\mathrm{core}}^{\alpha}$

and

$N_{\text {vir }}^{\alpha}=N^{\alpha}-N_{\text {docc }}^{\alpha}$,

respectively.

The above mentioned methods were implemented into the development version of the GAMESS program $[48,49]$, which was used in calculations for the next Section.

\section{Numerical assessments}

\subsection{DM-MP2 and FT-MP2 calculations of benzene}

First, the relation between FT-MP2 and DM-MP2, derived in the previous Section, was numerically assessed in calculations of benzene molecule with $6-31 \mathrm{G}^{* *}$ basis set [50]. In DM-MP2 calculations, we applied the Chebyshev expansion for the evaluation of matrix exponential, which is implemented in the EXPOKIT library program [51]. For the numerical quadrature of the Laplace-transformed integrals of Eqs. (9) and (27), we used the $\tau$-point Euler-Maclaurin (trapezoidal) quadrature

$$
\begin{aligned}
\int_{0}^{\infty} e_{2}(t) d t= & \int_{0}^{1} f_{2}(r) d r \\
\approx & \frac{1}{\tau+1}\left[\sum_{k=1}^{\tau} f_{2}\left(\frac{k}{\tau+1}\right)\right. \\
& \left.+\frac{f_{2}(0)+f_{2}(1)}{2}\right]
\end{aligned}
$$

with

$f_{2}(r)=e_{2}(t) \frac{d t}{d r}$

and the following change of variable

$t=\frac{r^{3}-0.9 r^{4}}{(1-r)^{2}}+r^{2} \tan \left(\frac{\pi r}{2}\right)$

according to the previous assessment [18] (note that $f_{2}(0)=$ $\left.f_{2}(1)=0\right)$.

Figure 1 shows the inverse temperature $(\beta)$ dependence of the FT-MP2 [Eqs. (21) and (25)] and the DM-MP2 [Eq. (27)] energies of a benzene molecule with $\mathrm{C}-\mathrm{C}$ and $\mathrm{C}-\mathrm{H}$ bond lengths of 140 and $109 \mathrm{pm}$. In the DM-MP2 calculations, the number of quadrature points was set to $\tau=7$. The MP2 energy at zero temperature $(-231.535$ Hartree $)$ is shown with dotted line. At low temperature ( $\beta \geq 30$ a.u.), all the four MP2 energies shown in Fig. 1 coincide with

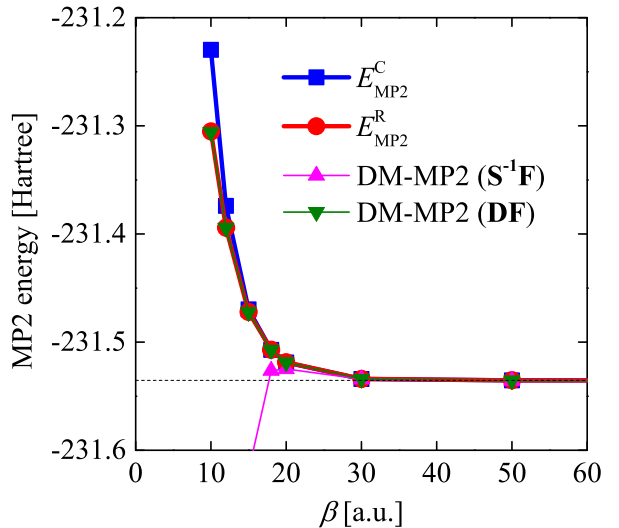

Fig. 1 Inverse temperature $(\beta)$ dependence of the FT- and DM-MP2 energies of benzene.

Table 1 The dependence of FT DM-MP2 energies (in Hartree) of benzene on the number of numerical quadrature points, $\tau .{ }^{\text {a) }}$

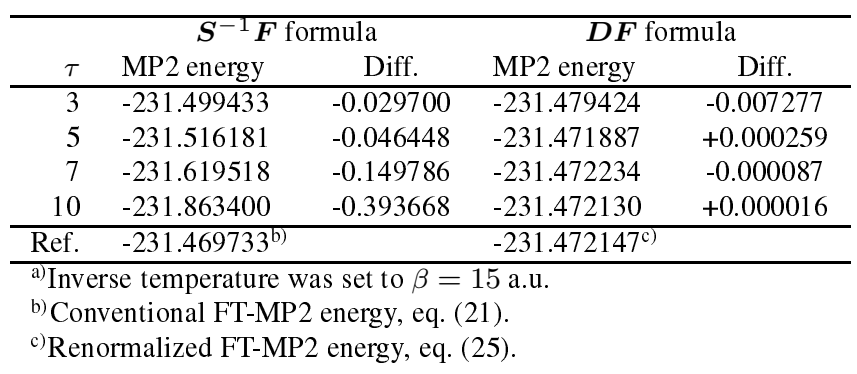

the zero-temperature energy because the benzene molecule has large band gap. For $\beta \leq 20$ a.u., the energy gradually increases as $\beta$ decreases, except for the DM-MP2 result with the $\boldsymbol{S}^{-1} \boldsymbol{F}$ formula. Even in this high-temperature region, the DM-MP2 result with $\boldsymbol{D} \boldsymbol{F}$ formula agrees well with the renormalized FT-MP2 one: the difference between these two results comes from the numerical quadrature error of Eq. (27). The DM-MP2 result with $\boldsymbol{S}^{-1} \boldsymbol{F}$ formula shows divergent behavior as $\beta$ decreases due to the divergent terms of Eq. (22). As described in Sect. 2.2, these divergent terms are not taken into account in the conventional FT-MP2 calculations. Although the conventionalFT-MP2 result shows good agreement with the renormalized FT-MP2 one up to $\beta=15$ a.u., the difference between two FT-MP2 results increases as $\beta$ decreases. In addition, this result numerically supports the reason for the divergence of DM-MP2 with $\boldsymbol{S}^{-1} \boldsymbol{F}$ formula when the density matrix is approximately obtained.

Table 1 shows the dependence of FT DM-MP2 energies of benzene on the number of numerical quadrature points, $\tau$. Here, the results for $\beta=15$ a.u., where two DM-MP2 energies in Fig. 1 show large discrepancy, are given. The energy with the $\boldsymbol{D F}$ formula smoothly converges to the renormalized FT-MP2 energy, $E_{\mathrm{MP} 2}^{\mathrm{R}}$, as $\tau$ increases. The energy with $\tau=7$, adopted in Fig. 1, only differs by $0.1 \mathrm{mHartree}$ from 
the renormalized FT-MP2 energy. On the contrary, the result with the $\boldsymbol{S}^{-1} \boldsymbol{F}$ formula shows divergent behavior as $\tau$ increases. This divergence may be a correct behavior because the FT DM-MP2 energy with $\boldsymbol{S}^{-1} \boldsymbol{F}$ formula includes the divergent terms, which are avoided when evaluating $E_{\mathrm{MP} 2}^{\mathrm{C}}$.

\subsection{Polyene system with bond alternation}

Next, we assessed the FT DC-MP2 method in calculations of polyene system, $\mathrm{C}_{60} \mathrm{H}_{62}$, with bond alternation, depicted in Fig. 2. $\Delta R_{\mathrm{BA}}$ indicates the magnitude of bond alternation, i.e., larger $\Delta R_{\mathrm{BA}}$ leads more single- and double-bond alternating picture and $\Delta R_{\mathrm{BA}}=0$ means fully delocalized structure. All the bond angles of $\angle \mathrm{C}-\mathrm{C}-\mathrm{C}$ and $\angle \mathrm{C}-\mathrm{C}-\mathrm{H}$ were set to be $120^{\circ}$. $\mathrm{A} \mathrm{HC}=\mathrm{CH}$ (or $\mathrm{H}_{2} \mathrm{C}=\mathrm{CH}$ for the edges) unit was adopted as the central region and its adjacent $n_{b}^{\mathrm{HF}}$ and $n_{b}^{\mathrm{MP} 2}$ units (on either side) were adopted as the buffer regions in DC-HF and DC-MP2 calculations, respectively.

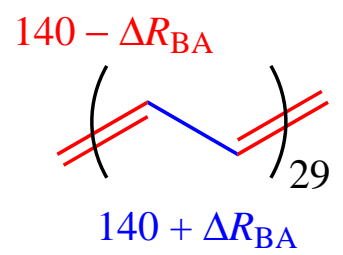

Fig. 2 Polyene system, $\mathrm{C}_{60} \mathrm{H}_{62}$, with bond alternation, $\Delta R_{\mathrm{BA}}$. Values in the Figure are the bond lengths in pm.

Figure 3 shows the dependence of the DC-MP2 energy deviation of polyene system, $\mathrm{C}_{60} \mathrm{H}_{62}$, from the zerotemperature canonical MP2 energy on the bond alternation, $\Delta R_{\mathrm{BA}}$. Three DC-MP2 formalisms (I for integer occupation, $\mathrm{C}$ for conventional FT, and $\mathrm{R}$ for renormalized FT) were used. In the MP2 correlation calculation, the $\mathrm{C} 1 \mathrm{~s}$ orbitals were frozen. We adopted two different inverse temperature parameters, i.e., (a) lower temperature $(\beta=500$ a.u.) and (b) higher temperature $(\beta=50$ a.u.). Here, the $6-31 \mathrm{G}^{* *}$ basis set [50] was used. The DC-HF buffer size used in these calculations was $n_{b}^{\mathrm{HF}}=6$. The energy deviations with $n_{b}^{\mathrm{MP} 2}=6$ (solid lines) are always smaller than with $n_{b}^{\mathrm{MP} 2}=4$ (dashed lines). For large $\Delta R_{\mathrm{BA}}$, the difference between the conventional and renormalized FT DCMP2 results is tiny: 0.14 mHartree or less for $\Delta R_{\mathrm{BA}}=4$ $\mathrm{pm}$. The difference between the integer-occupation and FTMP2 is also small for $\Delta R_{\mathrm{BA}}=10 \mathrm{pm}$, the maximum differences are 0.06 and $0.22 \mathrm{mHartree}$ with $\beta=500$ and 50 a.u., respectively. As the bond alternation, $\Delta R_{\mathrm{BA}}$, decreases, the energy deviation gradually increases because more delocalized electronic structure makes the DC approximation worse. The difference between the integer-occupation and FT-MP2 also increases: for $\Delta R_{\mathrm{BA}}=4 \mathrm{pm}$, the maximum differences are 0.7 and $1.5 \mathrm{mHartree}$ with $\beta=500$ and 50 a.u., respectively. For the same $\Delta R_{\mathrm{BA}}$ and $n_{b}^{\mathrm{MP} 2}$, the energy deviations obtained by the FT DC-MP2 formulas are smaller than those by the integer-occupation DC-MP2 one except for the renormalized FT-MP2 result with $\Delta R_{\mathrm{BA}}=0$, $\beta=50$ a.u., and $n_{b}^{\mathrm{MP} 2}=4$. Because the renormalized FTMP2 energy also shows divergent behavior for small bandgap systems, the conventional FT-MP2, in which the divergent terms are necessarily avoided, may be a better choice when the FT-MP2 formula is combined with the DC-MP2 method.


Fig. 3 Bond-alternation $\left(\Delta R_{\mathrm{BA}}\right)$ dependence of the DC-MP2 energy deviations of polyene system, $\mathrm{C}_{60} \mathrm{H}_{62}$, with integer occupation $\left(E_{\mathrm{MP} 2}^{\mathrm{I}}\right)$, conventional FT $\left(E_{\mathrm{MP} 2}^{\mathrm{C}}\right)$, and renormalized FT $\left(E_{\mathrm{MP} 2}^{\mathrm{R}}\right)$ formalisms for (a) $\beta=500$ a.u. and (b) $\beta=50$ a.u., where $\beta$ is the inverse temperature parameter appeared in the occupation number.

Table 2 summarizes the practical numbers of occupied and virtual MOs, defined by Eqs. (58) and (59), in the FT DC-MP2 calculations of $\mathrm{C}_{60} \mathrm{H}_{62}$ without bond alternation (i.e., $\Delta R_{\mathrm{BA}}=0$ ). The data for two characteristic subsystems, namely, the middle and edge subsystems, are provided in the Table. The inverse temperature parameter, $\beta$, was varied from 50 to 500 a.u. The numbers for the integeroccupation DC-MP2 are given together for comparison. The buffer size was fixed to $n_{b}^{\mathrm{HF}}=n_{b}^{\mathrm{MP} 2}=6$. Even for this de- 
Table 2 The practical numbers of occupied $\left(N_{\text {occ }}^{\alpha}\right)$ and virtual $\left(N_{\text {vir }}^{\alpha}\right)$ MOs for middle and edge subsystems in the FT DC-MP2 calculations of $\mathrm{C}_{60} \mathrm{H}_{62}$ with $\Delta R_{\mathrm{BA}}=0$. ${ }^{\text {a }}$

\begin{tabular}{llrrrrr}
\hline & & \multicolumn{5}{c}{$\beta$ [a.u.] } \\
Subsystem & & 50 & 100 & 200 & 500 & $\infty^{\mathrm{b}}$ \\
\hline Middle & $N_{\text {occ }}^{\alpha}$ & 128 & 99 & 72 & 68 & 66 \\
& $N_{\text {vir }}^{\alpha}$ & 474 & 434 & 429 & 428 & 428 \\
\multirow{4}{*}{ Edge } & $N^{\alpha}$ & 520 & 520 & 520 & 520 & 520 \\
& $N_{\text {occ }}^{\alpha}$ & 70 & 54 & 39 & 37 & 36 \\
& $N_{\text {vir }}^{\alpha}$ & 260 & 238 & 235 & 235 & 235 \\
& $N^{\alpha}$ & 285 & 285 & 285 & 285 & 285 \\
\hline${ }^{\mathrm{a}} n_{b}^{\mathrm{HF}}=n_{b}^{\mathrm{MP} 2}=6$. \\
${ }^{\mathrm{b}}$ Numbers for the integer-occupation DC-MP2 calculation.
\end{tabular}

localized system, the numbers for $\beta \geq 200$ a.u. are close to those for the integer-occupation DC-MP2 method. Therefore, in case of large $\beta$, the additional computational costs for the FT-MP2 treatment are tiny, although the energy improvement shown in Fig. 3 is considerable. $N_{\text {occ }}^{\alpha}$ and $N_{\text {vir }}^{\alpha}$ gradually increases as the temperature increases. It was concluded that the use of low temperature is important in DCMP2 calculation not only to improve the accuracy but also to reduce the computational demands for the FT-MP2 treatment. Although the use of high temperature often improves the self-consistent field convergence in DC-HF calculation, so-called annealing technique can be adopted to lower the final temperature [52].

Up to this point, as discussed in Sect. 2.3, the Fermi level determined in the DC-HF calculation was also used for Eqs. (54) and (55). If the Fermi level is redetermined using the subsystem MOs constructed for the DC-MP2 calculation and is used for Eqs. (54) and (55), the result of Fig. 3 changes to be Fig. 4 for $\beta=500$ a.u. Here, the data for $n_{b}^{\mathrm{MP} 2}=6$ are not given, because the result does not change for the case of $n_{b}^{\mathrm{HF}}=n_{b}^{\mathrm{MP} 2}$. For the integer-occupation DCMP2 method, the results of Fig. 3 (a) and 4 are the same. On the other hand, the results for the FT DC-MP2 calculations are considerably different between Figs. 3 (a) and 4. For example, the differences between the integer-occupation and the conventional FT DC-MP2 energies for $\Delta R_{\mathrm{BA}}=0$ are 1.5 and 16.7 mHartree for Figs. 3 (a) and 4, respectively. The renormalized FT DC-MP2 result shows divergent behavior at $\Delta R_{\mathrm{BA}}=0$, as was also observed in Fig. 3 (b). Although the detailed analysis may be required for the optimal determination of the Fermi level used in the DC-MP2 calculation, the authors use the Fermi level determined in the DC-HF calculation hereafter.

Finally, the present FT DC-MP2 results are compared with the DC-DM MP2 one, where the correlation energy is obtained from Eq. (9) with Eqs. (16), (17), and the DC$\mathrm{HF}$ density matrices. Figure 5 shows the buffer-size dependence of the FT DC-MP2 and DC-DM MP2 energy deviations of polyene system, $\mathrm{C}_{60} \mathrm{H}_{62}$, with $\Delta R_{\mathrm{BA}}=0$. The inverse temperature was fixed at $\beta=500$ a.u. In the DC-

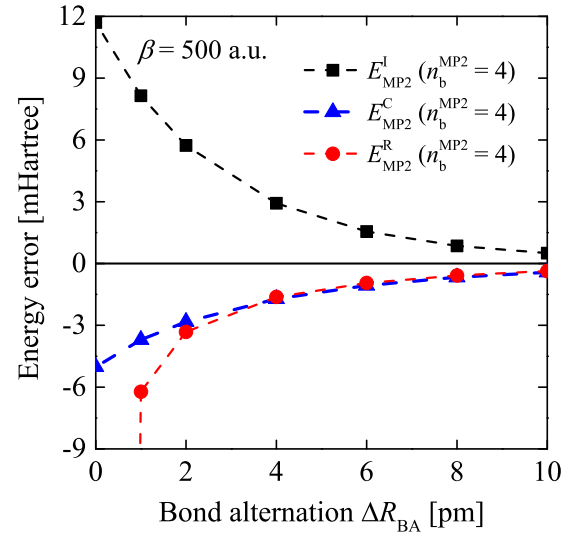

Fig. 4 The same figure as Fig. 3 (a) but for the case that the Fermi level, $\varepsilon_{\mathrm{F}}$, is determined using the subsystem MOs reconstructed for the DC-MP2 calculation with smaller buffer size.

DM MP2 calculations, the number of quadrature points was set to $\tau=7$. For comparing with the DC-DM MP2 result, the computational details used in calculations for Fig. 5 are different from those for Fig. 3: the buffer region was fixed throughout the DC-HF and DC-MP2 calculations, i.e., $n_{b}^{\mathrm{HF}}=n_{b}^{\mathrm{MP} 2}$, the core electrons were correlated as well as the valence ones (i.e., $N_{\text {core }}^{\alpha}=0$ for all subsystems $\alpha$ ), and the 6-31G basis set [53] was used. The numerical quadrature scheme used in DC-DM MP2 calculations is the same as used in the DM-MP2 calculations of benzene, mentioned in Sect. 3.1. The integer-occupation DC-MP2 results are also shown for comparison. Among three subsystem-based DCMP2 results, the conventional FT-MP2 method shows the best agreement with the zero-temperature canonical MP2 result, although the DC-DM MP2 result gives smaller energy deviation than the subsystem-based DC-MP2 method. The renormalized FT-MP2 results are also improved from the integer-occupation DC-MP2 when adopting the same buffer region for DC-HF and DC-MP2 calculations. The authors again concluded that the use of FT-MP2 formulas with low electronic temperature improves the accuracy of the DC-MP2 calculations especially with the conventional FT-MP2 formalism with slight addition of the computational demands, although a moderate improvement can also be confirmed with the renormalized FT-MP2 formalism when the same buffer region is adopted for DC-HF and DC-MP2 calculations.

\section{Conclusion}

It was found that two different representations of densitymatrix (DM) MP2, which Surján originally formulated for pure state $(\beta=\infty)$ [14], can be obtained from the Laplace transformation of two types of finite-temperature (FT) MP2 formulas, namely, $\boldsymbol{S}^{-1} \boldsymbol{F}$ formula from the conventional 


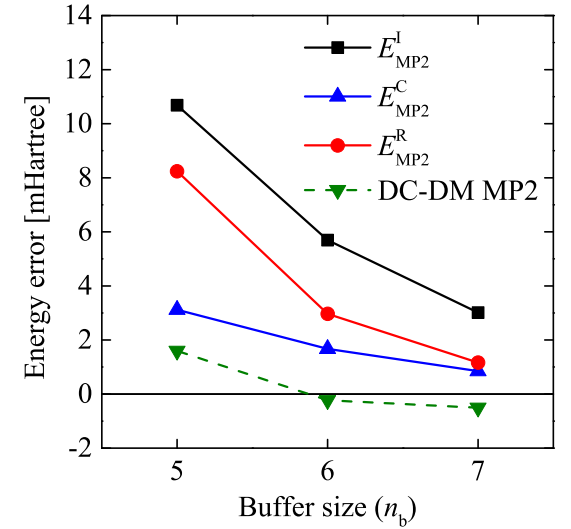

Fig. 5 Buffer-size $\left(n_{b}=n_{b}^{\mathrm{HF}}=n_{b}^{\mathrm{MP} 2}\right)$ dependence of the FT DCMP2 and DC-DM MP2 energy deviations of polyene system, $\mathrm{C}_{60} \mathrm{H}_{62}$, at $\Delta R_{\mathrm{BA}}=0$ and $\beta=500$ a.u. 6-31G basis set was adopted.

FT-MP2 and $\boldsymbol{D} \boldsymbol{F}$ formula from the renormalized FT-MP2. We numerically confirmed this one-to-one correspondence of FT-MP2 and DM-MP2 for benzene molecule by varying the electronic temperature $\beta$ and found that the DMMP2 energy with $\boldsymbol{S}^{-1} \boldsymbol{F}$ formula shows unfavorable behavior due to the divergent term. This fact also accounts for our previous experience [18] that the DM-MP2 calculation with $\boldsymbol{S}^{-1} \boldsymbol{F}$ formula fails to obtain approximate MP2 energy when the density matrix is approximated. We also applied the FT-MP2 energy to the subsystem MO-based divide-andconquer (DC) MP2 method. The FT DC-MP2 energy shows better agreement with the zero-temperature canonical MP2 energy than the previous integer-occupation DC-MP2 one, in spite of its tiny additional computational efforts especially for large $\beta$. For the combination of FT-MP2 and DC-MP2 method, the use of the conventional FT-MP2 formalism that directly avoid the divergent terms may be more preferable than that of the renormalized FT-MP2.

Acknowledgements The authors are grateful to Prof. Hiromi Nakai and Dr. Takeshi Yoshikawa (Waseda University) for their valuable comments. Some of the present calculations were performed using the computer facilities at Research Center for Computational Science, Okazaki and at Research Institute for Information Technology, Kyushu University, Japan. This work was supported in part by JSPS KAKENHI Grant Number 25810011.

\section{References}

1. Helgaker T, Jørgensen P, Olsen J (2002) Molecular ElectronicStructure Theory. Wiley, Chichester

2. Rolik Z, Szabados Á, Surján PR (2003) J Chem Phys 119:1922

3. Szabados Á, Rolik Z, Tóth G, Surján PR (2005) J Chem Phys 122:114104

4. Surján P, Rolik Z, Szabados Á, Kőhalmi D (2004) Ann Phys 13:223
5. Kobayashi M, Szabados Á, Nakai H, Surján PR (2010) J Chem Theory Comput 6:2024

6. Szabados Á, Nagy P (2011) J Phys Chem A 115:523

7. Surján PR (1999) An Introduction to the Theory of Geminals. In: Surján PR (ed) Correlation and Localization. Springer, Berlin, pp. 63-88

8. Jeszenszki P, Nagy PR, Zoboki T, Szabados Á, Surján PR (2014) Int J Quantum Chem 114:1048

9. Tarumi M, Kobayashi M, Nakai H (2012) J Chem Theory Comput $8: 4330$

10. Møller C, Plesset MS (1934) Phys Rev 46:618

11. Shavitt I, Bartlett RJ (2009) Many-Body Methods in Chemistry and Physics: MBPT and Coupled-Cluster Theory. Cambridge University Press, Cambridge

12. Tsuneda T (2014) Density Functional Theory in Quantum Chemistry. Springer, Tokyo

13. Engel E, Dreizler RM (2011) Density Functional Theory: An Advanced Course. Springer, Heidelberg

14. Surján PR (2005) Chem Phys Lett $406: 318$

15. Almlöf J (1991) Chem Phys Lett 181:319

16. Häser M (1993) Theor Chim Acta 87:147

17. Ayala PY, Scuseria GE (1999) J Chem Phys 110:3660

18. Kobayashi M, Nakai H (2006) Chem Phys Lett 420:250

19. Surján PR, Szabados Á (2011) Perturbative Approximations to Avoid Matrix Diagonalization. In: Papadopoulos MG, Zalesny R, Mezey PG, Leszczynski J (eds) Linear-Scaling Techniques in Computational Chemistry and Physics: Methods and Applications. Springer, Dordrecht, pp. 83-95

20. Kobayashi M, Nakai H (2011) Divide-and-Conquer Approaches to Quantum Chemistry: Theory and Implementation. In: Papadopoulos MG, Zalesny R, Mezey PG, Leszczynski J (eds) Linear-Scaling Techniques in Computational Chemistry and Physics: Methods and Applications. Springer, Dordrecht, pp. 97127

21. Kobayashi M, Nakai H (2012) Phys Chem Chem Phys 14:7629

22. Akama T, Kobayashi M, Nakai H (2007) J Comput Chem 28:2003

23. Yang W (1991) Phys Rev Lett 66:1438

24. Yang W, Lee TS (1995) J Chem Phys 103:5674

25. Kobayashi M, Akama T, Nakai H(2006) J Chem Phys 125:204106

26. Kobayashi M, Imamura Y, Nakai H (2007) J Chem Phys 127:074103

27. Kobayashi M, Nakai H (2008) J Chem Phys 129:044103

28. Kobayashi M, Nakai H (2009) J Chem Phys 131:114108

29. Yoshikawa T, Kobayashi M, Nakai H (2013) Int J Quantum Chem 113:218

30. Bloch C (1965) Diagram Expansions in Quantum Statistical Mechanics. In: de Boer J, Uhlenbeck GE (eds) Studies in Statistical Mechanics, Vol. 3. North-Holland, Amsterdam, pp. 3-211

31. Blaizot JP, Ripka G (1985) Quantum Theory of Finite Systems. The MIT Press, Cambridge

32. Hirata S, He X (2013) J Chem Phys 138:204112

33. Kohn W, Luttinger JM (1960) Phys Rev 118:41

34. Matsubara T (1955) Prog Theor Phys 14:351

35. Thouless DJ (1957) Phys Rev 107:1162

36. Cremer D (2011) WIREs Comput Mol Sci 1:509

37. Lipparini E (2008) Modern Many-Particle Physics, 2nd edn. World Scientific, Singapore

38. Thouless DJ (1972) The Quantum Mechanics of Meny-Body Systems, 2nd edn. Academic Press, New York

39. Cohen AJ, Mori-Sánchez P, Yang W (2009) J Chem Theory Comput 5:786

40. Mattuck RD (1976) A Guide to Feynman Diagrams in the ManyBody Problem. McGraw-Hill, New York

41. Yoshikawa T, Kobayashi M, Nakai H (2011) Theor Chem Acc 130:411

42. Yoshikawa T, Nakai H (2015) Theor Chem Acc 134:53

43. Mezey PG (1995) J Math Chem 18:141 
44. Szekeres Z, Mezey PG, Surján PR (2006) Chem Phys Lett 424:420

45. Nakai H (2002) Chem Phys Lett $363: 73$

46. Kobayashi M, Nakai H (2009) Int J Quantum Chem 109:2227

47. Kobayashi M, Nakai H (2013) J Chem Phys 138:044102

48. Schmidt MW, Baldridge KK, Boatz JA, Elbert ST, Gordon MS, Jensen JH, Koseki S, Matsunaga N, Nguyen KA, Su S, Windus TL, Dupuis M, Montgomery JA Jr (1993) J Comput Chem 14:1347

49. Gordon MS, Schmidt MW (2005) Advances in Electronic Structure Theory: GAMESS a Decade Later. In: Dykstra CE, Frenking G, Kim KS, Scuseria GE (eds) Theory and Applications of Computational Chemistry: The First Forty Years. Elsevier, Amsterdam, pp. $1167-1189$

50. Hariharan PC, Pople JA (1973) Theor Chim Acta 28:213

51. Sidje RB (1998) ACM Trans Math Software 24:130

52. Akama T, Kobayashi M, Nakai H (2009) Int J Quantum Chem 109:2706

53. Hehre WJ, Ditchfield R, Pople JA (1972) J Chem Phys 56:2257 